\title{
Genetic Testing for Lung Cancer Risk
}

\author{
If Physicians Can Do It, Should They? \\ Theodore W. Marcy, MD, MPH, Michael Stefanek, PhD, Kimberly M. Thompson, ScD
}

\begin{abstract}
Advances in genetics have increased our ability to assess an individual's genetic risk for disease. There is a hypothesis that genetic test results will motivate high-risk individuals to reduce harmful exposures, to increase their surveillance for disease, or to seek preventive treatments. However, genetic testing for genes associated with an increased risk of lung cancer would not change physicians' recommendations regarding smoking cessation. Limited studies suggest that test results that demonstrate an increased risk of lung cancer do not improve smoking cessation success. These test results may even distort an individual's risk perceptions. Before recommending genetic testing to assess risk for disease, physicians need to consider whether knowledge about genetic susceptibility will alter patient management.
\end{abstract}

KEY WORDS: lung neoplasms; genetic screening; genetic counseling; smoking.

J GEN INTERN MED 2002;17:946-951.

$\mathbf{O}$ ur advancing knowledge about the human genome will allow us to test individuals for increased genetic susceptibility to a variety of diseases. Genetic testing could encourage high-risk individuals to reduce their harmful exposures, to increase their surveillance for early disease, or to consider preventive therapy. ${ }^{1}$ As an example, women who have BRCA 1 and 2 mutations have an increased lifetime risk of breast cancer as high as $85 \%$ compared to that of $12 \%$ in the general population of women. In addition to the recommendations for women in general, these highrisk women have been advised to avoid hormonal replacement therapy, to undergo annual mammography and annual or semiannual clinical breast examinations beginning at age 25 to 35 years, to consider prophylactic

Received from the National Cancer Institute, Division of Cancer Prevention, Office of Preventive Oncology (TWM), and the National Cancer Institute, Division of Cancer Control and Population Sciences, Basic Biobehavioral Research Branch (MS), Rockville, Md; the Office of Health Promotion Research, University of Vermont College of Medicine and the Vermont Cancer Center, University of Vermont (TWM), Burlington, Vt; and the Department of Health Policy and Management, Harvard School of Public Health (KMT), Boston, Mass.

Address correspondence and requests for reprints to Dr. Marcy: Office of Health Promotion Research, University of Vermont, One South Prospect St., Burlington, VT 05401 (e-mail: theodore.marcy@uvm.edu). bilateral mastectomy, ${ }^{2}$ or to consider use of Tamoxifen as a chemo preventive agent. ${ }^{3,4}$ The information from genetic testing could also provide women with a strong family history of breast cancer with more accurate assessments of their personal risk of cancer, and reduce their uncertainty, ${ }^{5}$ although such testing must be combined with skilled preand post-test counseling.

Identifying genetic susceptibility in other circumstances, however, may not have this potential clinical value, and may actually contribute to inaccurate perceptions of risk. The health care community should assess whether information from genetic testing would actually change the clinical recommendations of the health care provider, or the behavior of an individual to be tested, before these tests are used to estimate an individual's risk. To explore this, we consider the specific situation of using genetic testing to determine whether an individual is at increased risk of lung cancer from smoking.

\section{EVIDENCE FOR GENETIC FACTORS AS A DETERMINANT IN LUNG CANCER RISK}

Smoking is estimated to cause $87 \%$ of all lung cancers in the United States, including 90\% of the cases in men and $79 \%$ of the cases in women. ${ }^{6}$ However, the cumulative incidence of lung cancer is only $14 \%$ in males who smoke more than a pack a day of cigarettes and survive to age $74 .^{7}$ Thus, lung cancer is the cause of death in only a minority of cigarette smokers, suggesting that there are host-specific factors that influence susceptibility to lung cancer, not unlike many human diseases.

Multistage models of carcinogenesis provide a conceptual framework for understanding the associations of lung cancer incidence with smoking intensity, duration, and smoking cessation. ${ }^{8}$ These models propose that carcinogens derived from chemical components in tobacco smoke react with the DNA of respiratory epithelial cells. Some of the carcinogens initiate mutagenic changes while others promote the growth of these mutated cells or disable genes that suppress tumor growth. Mutagenesis, growth promotion, and inhibition of tumor suppression may all be necessary for clinically evident cases of lung cancer to develop. This may explain the salutary effects of smoking cessation on lung cancer incidence even after years of tobacco use.

Procarcinogens in tobacco smoke must be metabolically transformed in order to exert their carcinogenic 
effect. ${ }^{9}$ At the same time, other enzymes detoxify these chemicals into inactive compounds, thus creating a dynamic equilibrium of carcinogen concentration in lung tissues for any given level of tobacco smoke inhalation. Genetic polymorphisms-slight differences in a gene's nucleotide sequence-produce slightly different forms of the enzyme, resulting in variations in enzyme kinetics. These inherited differences in the transformation rate of environmental substances are one source of variability in disease risk within a population.

For example, the enzyme CYP2D6 can transform polycyclic aromatic hydrocarbons and arylamines into reactive chemical species that can bind covalently to DNA and induce mutations. About $90 \%$ to $95 \%$ of the population has CYP2D6 enzymes that are extensive or intermediate metabolizers, as measured by the speed with which they metabolize the drugs Debrisoquine and Dextromethorphan; the other $5 \%$ to $10 \%$ of the population has enzymes that are slow metabolizers. ${ }^{9,10}$ Extensive and intermediate metabolizers would be expected to have higher concentrations of these carcinogens in the lung than would slow metabolizers. The extensive metabolizing phenotype was associated with more than double the risk of lung cancer (odds ratio [OR], 2.28; 95\% confidence interval [95\% CI], 1.60 to 3.40 ) in a meta-analysis of 7 case-control studies comparing the enzymatic rate of CYP2D6 in lung cancer patients to that of matched controls. ${ }^{9}$ Polymerase chain reaction tests can now determine the actual CYP2D6 genotype in all but an estimated 10\% of slow metabolizers, making it easier to determine an individual's probable CYP2D6 enzyme kinetics. However, external factors, including age and certain medications, can alter CYP2D6 enzyme kinetics so that genotyping may not predict an individual's metabolic phenotype or their response to medications that are metabolized by this enzyme. ${ }^{11,12}$

The presence of an unfavorable or favorable gene allele does not change the probability of lung cancer in an individual to the extremes of either zero or one hundred percent. Uncertainty persists; the added information from genetic testing only changes the likelihood of lung cancer by some degree in one direction or the other. For example, since the slow-metabolizer genotype frequency in the population is only $6.4 \%,{ }^{10}$ then the lifetime risk of lung cancer in the extensive and intermediate metabolizers is approximately that of population estimates-about $14 \%{ }^{7}$ If extensive and intermediate metabolizers have double the risk of slow metabolizers, ${ }^{9}$ the lifetime risk of lung cancer in the slow metabolizers is approximately half of that, or about $7 \%$.

\section{KNOWLEDGE ABOUT GENETIC RISK AND BEHAVIOR CHANGE}

The behavior change recommended for smokers with genes that increase cancer susceptibility is very clear and unequivocal-smoking cessation. Switching to low-tar cigarettes does not reduce risk, so this is not a valid option. ${ }^{13}$ Numerous observational studies utilizing both case-control and cohort designs demonstrate that smoking cessation decreases the risk for lung cancer by $20 \%$ to $90 \%$ compared to current smokers, depending on the duration of abstinence. ${ }^{8}$ Those who are abstinent for 15 years have a $90 \%$ risk reduction, but their risk remains higher than that of never smokers. ${ }^{8}$

Physicians may expect that telling people that they have an increased genetic risk for lung cancer would increase their rate of smoking cessation. The effect of knowledge about genetic risk on health behavior, however, is complex and uncertain. There is only limited evidence of increased motivation, and no evidence of successful behavior change in the few studies that observed individuals who were told that they have increased genetic risk. ${ }^{14-16}$ This information may actually reduce motivation because it may induce a sense of powerlessness and fatalism. ${ }^{5,17}$

Smokers informed that they had an unfavorable genetic polymorphism might be expected to have heightened perceived risk and emotional responses that would increase motivation to quit. ${ }^{18}$ Investigators tested this hypothesis in a randomized controlled study that randomly assigned 427 smokers to 1 of 3 cessation treatments: minimal counseling, minimal counseling plus biofeedback on exhaled carbon monoxide, and minimal counseling plus biofeedback plus genotyping for CYP2D6. ${ }^{15,16}$ Those smokers who had the extensive metabolizing genotype were told:

\begin{abstract}
Because of your genetic make-up, you have the ability to activate chemicals in tobacco smoke into very dangerous cancer-causing substances. This means that your risk for getting lung cancer is not only higher than nonsmokers, but also may be higher than other smokers who do not have the same genetic make-up. ${ }^{15}$
\end{abstract}

The 11 smokers who were homozygous for the lowsusceptibility (poor metabolizer) CYP2D6 allele were excluded from the analysis, but still underwent treatment. They were told:

You do not have the ability to activate specific chemicals in tobacco smoke. However, you may still be at risk for lung cancer because your body may be able to use other ways to activate cancer-causing chemicals. ${ }^{15}$

The smokers with increased susceptibility in the genetic feedback group were more than twice as likely to make quit attempts than those in the other treatment groups (OR, 2.13; 95\% CI, 1.13 to 4.01), but were no more successful at long-term quitting. Similar negative results were recently reported in a study that randomized currently smoking low-income African Americans to free nicotine replacement therapy and self help material with or without genetic testing. ${ }^{19}$ There was no significant difference in the 12-month cessation rates between the 2 groups.

\section{SCREENING AND EARLY INTERVENTION STRATEGIES}

Attempts to detect early lung cancer by using chest radiographs with or without sputum cytology were not 
successful in improving the outcome from lung cancer in several randomized controlled trials. ${ }^{20,21}$ Low-dose spiral chest computed tomography (spiral CT) is a more sensitive technique for detecting early lung cancer, ${ }^{22}$ but has not yet been demonstrated to improve outcome from lung cancer. ${ }^{23,24}$ Studies evaluating the efficacy of spiral CT scans for screening are planned. No effective chemopreventive therapies are available for lung cancer, and the use of $\beta$-carotene was actually associated with a slight increase of risk for lung cancer in smokers. ${ }^{25}$ Prophylactic surgery is obviously not feasible. Therefore, secondary prevention strategies (early detection or treatment of disease) are not yet available to those individuals identified as being at greater risk of lung cancer.

\section{PUTTING THE RISK FOR LUNG CANCER IN PERSPECTIVE}

Physicians need to help patients put the perception of risk from lung cancer, regardless of the results of genetic testing, into proper perspective. People who smoke tend to focus on their risk of lung cancer because they associate lung cancer with more dread, or they have been told they have a 10 - to 20-fold greater risk for lung cancer than do nonsmokers. Current smokers need to know that there are many ways that smoking can kill them besides lung cancer, including cardiovascular disease, cerebrovascular disease, chronic lung diseases, and primary cancers of other organs. A 45-year-old man who smokes is more likely to die from premature cardiovascular disease than from lung cancer. ${ }^{26}$

The inaccurate perception that the risk of lung cancer exceeds that of cardiovascular disease is related to misunderstanding the differences between absolute risks, relative risks, and risk differences. The absolute risk is the risk of death in a population of people over a given period of time. A relative risk gives the magnitude and direction of change in disease incidence in those who have an exposure, expressed as multiples of the baseline risk of those not exposed. Because the absolute risk for lung cancer is very low in nonsmokers, the relative risk in those who do smoke is quite high, even though only about $14 \%$ of those smoking more than a pack a day die of lung cancer. The absolute risk of cardiovascular deaths in nonsmokers is, by contrast, quite high (Table 1). The risk difference (excess deaths related to smoking) is defined as the difference in death rate in those exposed as opposed to those not exposed. Smoking increases the relative risk of cardiovascular disease and sudden death by only 2 - to 4-fold, as opposed to the 10- to 20-fold increase risk of lung cancer. However, since the baseline absolute risk of death from cardiovascular disease in nonsmokers is high, multiplying this death rate by the relative risk of 2 to 4 makes the excess number of deaths from cardiovascular disease much higher than the excess number of deaths due to lung cancer from smoking.

Mattson et al. calculated the absolute risks of death in men from lung cancer, cardiovascular disease, and all
Table 1. Estimated Death Rates per 100,000 Male Population by Smoking Status, Selected Causes

\begin{tabular}{lccr}
\hline \hline $\begin{array}{l}\text { Cause of Death in 45- } \\
\text { to 54-year-old Men (1982) }\end{array}$ & $\begin{array}{c}\text { Never } \\
\text { Smoked }\end{array}$ & $\begin{array}{c}\text { Current <25 } \\
\text { Cigarettes/Day }\end{array}$ & $\begin{array}{r}\text { Former } \\
\text { Smoker }\end{array}$ \\
\hline Lung cancer & 7.0 & 67.0 & 43.5 \\
Coronary heart disease & 54.8 & 308.0 & 118.4 \\
$\begin{array}{l}\text { All smoking-related } \\
\quad \text { diseases }\end{array}$ & 68.5 & 445.6 & 192.2 \\
All causes & 389.6 & 923.4 & 518.2 \\
\hline
\end{tabular}

Adapted with permission from the American Public Health Association. ${ }^{26}$

causes for light smokers, heavy smokers, former smokers, and nonsmokers using aggregate data from the National Health Interview Survey and from the U.S. Veterans Study. ${ }^{26}$ Their calculations of absolute risk (deaths per 100,000) for 45- to 54-year-old males are reproduced in Table 1. In 45- to 54-year-old men who smoke about a pack a day ( $<25$ cigaretts/day) the risk difference for death from all causes compared to nonsmokers is 533.8 per 100,000. (For those who smoke $\geq 25$ cigarettes/day, the risk difference rises to 962.4 per 100,000.) Lung cancer accounts for only 60 of these excess deaths per 100,000 . By contrast, coronary heart disease accounts for 253.2 of the excess deaths-more than 4 times that for lung cancer.

Physicians should also acknowledge that smoking reduces health-related quality of life and can have adverse health impacts on family members. Exposure to environmental tobacco smoke (second-hand smoke) increases the frequency of respiratory tract disorders, including infection, ${ }^{27}$ asthma, ${ }^{28}$ and sudden infant death syndrome. ${ }^{29}$ Cigarette smoke may also increase a spouse's risk of developing lung cancer ${ }^{30}$ and cardiovascular disease. ${ }^{31}$

\section{THE HEALTH BENEFITS OF QUITTING}

Physicians should convince their patients who smoke that smoking cessation would reduce their risk of dying from a smoking-related cause regardless of the genetic testing results. The risk of death for former smokers from all causes is almost half that of current pack-a-day smokers (518.2/923.4 or 56\%), although it still exceeds that of nonsmokers (Table 1). The estimated absolute risk reduction associated with smoking cessation is 405.2 deaths per 100,000 . This is calculated by subtracting the death rate from all causes in former smokers from the death rate from all causes in current smokers, or 923.4 - 518.2 per 100,000 .

These data and this estimate reflect the current mixture in the population of underlying genetic susceptibility for disease. The risks for individuals with a CYP2D6 slow-metabolizer genotype may be expected to have a relatively lower risk of getting lung cancer, and consequently, their relative benefits from smoking cessation 
might be lower than the estimate of 405.2 per 100,000 . Similarly, the lifesaving benefits to the intermediate and extensive metabolizers might be higher, but with respect to the overall difference resulting from smoking cessation in the reduction of the risk of dying, these differences are small. For example, if we make the extreme assumption that a 45-year-old male with a CYP2D6 slow-metabolizer genotype experiences no lung cancer risk, then the reduction in his risk of dying associated with smoking cessation would be 381.7 per 100,000 (i.e., (923.4 - 67.0) $-(518.2-43.5)=381.7)$. Now, let us assume that CYP2D6 slow metabolizers represent $10 \%$ of the population, ${ }^{10}$ that they again have zero risk for lung cancer, and that $90 \%$ of the population are intermediate or extensive metabolizers. For the extensive and intermediate metabolizers, the estimated reduction in the overall risk of death associated with smoking cessation would be approximately 407.8 per 100,000 (calculated as $(405.2$ - 381.7p)/(1 - p) where $\mathrm{p}$ represents the percentage of the population who are slow metabolizers. Table 1 does not address the effects of smoking on quality of life or the adverse impact of environmental tobacco smoke on family members.

\section{THE VALUE OF GENETIC TESTING FOR LUNG CANCER RISK FROM SMOKING}

In the case of lung cancer risk assessment, the value of information from genetic testing for CYP2D6 or any gene allele associated with lung cancer is relatively limited because the recommendation to a current smoker who undergoes testing remains the same regardless of the results-advise them to quit, assist them in their quit attempt with counseling and pharmacotherapy, and arrange follow-up for cessation attempts. No additional screening or chemo-preventive agents are, at this time, indicated.

Physicians can be effective agents for health behavior change because they see $70 \%$ of the 46.5 million current smokers in the United States each year. Even if a physician's brief advice increases the long-term quit rate by only an additional $2 \%$ per year, this translates into 930,000 additional former smokers each year. Physician smoking cessation interventions compare favorably with almost any other physician intervention estimated in economic analyses, ${ }^{32}$ with a cost of $\$ 3,539$ per life-yearsaved. By comparison, screening for hypertension costs $\$ 23,335$ per life-year-saved. ${ }^{32}$

It is a challenge to help current smokers perceive the health risks of smoking accurately. Smokers tend to selectively retain information that reduces the tension of continuing a valued and enjoyed habit that is also hazardous. ${ }^{33}$ After all, smoking is performed many times a day without immediate adverse health consequences, and smoking enhances the subjective sense of well-being temporarily by reducing anxiety and withdrawal symptoms. These experiences may distort the perceived risk so that it is lower than the actual risk. ${ }^{34}$ Genetic test results that indicate a reduced risk of lung cancer may further distort an individual's falsely low perceived risk. Results that suggest an individual has an increased risk may be wrongly interpreted to imply inevitability, reducing the motivation to quit. ${ }^{35}$ At present, there is no information on whether a test result that implies a decreased lung cancer risk will reduce the number of subsequent quit attempts by current smokers. If testing does reduce quit attempts, it could actually have adverse health effects.

\section{WHEN IS GENETIC TESTING INDICATED FOR PREVENTION?}

The Genetics Working Group of the Centers of Disease Control and Prevention listed 8 issues to consider when developing policies for genetic testing in disease prevention (Table 2). ${ }^{1}$ By these criteria, genetic testing for lung cancer risk might be appropriate if there were an accurate, reasonably priced test. First, lung cancer has a major public health impact that will cause an estimated 154,900 deaths in the United States in 2002. ${ }^{36}$ Second, the genotype of increased risk is prevalent, with $90 \%$ to $95 \%$ of the population having the genotype associated with increased risk. Laboratory quality issues (the third criterion) and cost (the seventh criterion) are difficult to address, because genotyping for CYP2D6 is not clinically available. The magnitude of the association of the genotype and lung cancer (the fourth criterion) has not been prospectively verified. Although case-control studies observed a 2-fold increase in risk for lung cancer with the high-risk phenotype, genotyping was not done, and therefore these studies could not determine whether the genotype is associated with lung cancer.

Smoking cessation is available as a preventive method since smoking is known to be a modifiable risk factor for disease (criteria 5 and 6). However, the relationship of this modifiable risk factor-smoking-to other diseases with major public health impact is missing from these criteria. If

\section{Table 2. Issues to Consider in Using Genetic Testing in Disease Prevention}

1. Public health impact of disease: incidence, prevalence, morbidity, mortality

2. Prevalence of genotype

3. Laboratory quality issues: analytic sensitivity, specificity, and predictive values of genetic test

4. Magnitude of association between genotype and disease: relative, absolute, and attributable risks

5. Interaction with known modifiable risk factors for the disease

6. Available intervention or prevention methods

7. Cost of test

8. Ethical, legal, and social issues

Reprinted with permission from the American Public Health Association. $^{1}$ 
the risk of a modifiable risk factor is far greater than the risk conferred by a genetic factor, then the value of genetic testing is low. This would be true even in the hypothetical situation in which genotyping was accurate (criterion 3), associated with lung cancer risk in prospective studies (criterion 4), and inexpensive (criterion 7). In our example, genetic testing does not change our uncompromising recommendation-to advise patients not to smoke regardless of the genetic test results. In other selected diseases, genetic testing can provide highly valuable information. When a test does have the potential for benefit, then a physician's recommendations for testing must weigh ethical, legal, and social issues (criterion 8), such as cost, confidentiality, access to testing, and appropriate follow-up care. If the patient believes that the test information would be of value to them irrespective of the larger societal issues, then health care providers should carefully consider testing on a case-by-case basis.

Physicians need to consider the value of the information from a genetic test before recommending that test to patients. From an ethical, legal, and societal perspective, it is difficult to support testing that has no demonstrated positive effect on health outcomes. If genetic testing will not alter patient management, then recommending this testing may not be appropriate medical care.

This work was supported by the National Cancer Institute Cancer Prevention Fellowship Program.

\section{REFERENCES}

1. Khoury M. From genes to public health: the applications of genetic technology in disease prevention. Am J Public Health. 1996;86: 1717-22.

2. Hartmann L, Sellers T, Schaid D, et al. Efficacy of bilateral prophylactic mastectomy in BRCA1 and BRCA2 gene mutation carriers. J Natl Cancer Inst. 2001;93:1633-7.

3. Burke W, Daly M, Garber J, Botkin J, Kahn M, Lynch P. Recommendations for follow-up care of individuals with an inherited predisposition to cancer. II. JAMA. 1997;277:997-1003.

4. Stefanek M, Hartmann L, Nelson W. Risk-reduction mastectomy: clinical issues and research needs. J Natl Cancer Inst. 2001;93: 1297-306.

5. Marteau T, Croyle R. Psychological responses to genetic testing. BMJ. 1998;316:693-6.

6. Wingo P, Ries L, Giovino G, et al. Annual report to the nation on the status of cancer, 1973-1996, with a special section on lung cancer and tobacco smoking. J Natl Cancer Inst. 1999;91:675-90.

7. Samet J, Wiggins C, Humble C, Pathak D. Cigarette smoking and lung cancer in New Mexico. Am Rev Respir Dis. 1988;137: 1110-3.

8. Public Health Service, Centers for Disease Control, Center for Chronic Disease Prevention and Health Promotion, Office on Smoking and Health. The Health Benefits of Smoking Cessation. Washington, DC: U.S. Department of Health and Human Services; 1990. DHHS Publication No. (CDC) 90-8416.

9. Amos C, Caporaso N, Weston A. Host factors in lung cancer risk: a review of interdisciplinary studies. Cancer Epidemiol Biomarkers Prev. 1992;1:505-13.

10. Steiner E, Iselius L, Alvan G, Lidnsten J, Sjoqvist F. A family study of genetic and environmental factors determining polymorphic hydroxylation of debrisoquin. Clin Pharmacol Ther. 1985;38: 394-401.

11. Scordo M, Spina E. Cytochrome P450 polymorphisms and response to antipsychotic therapy. Pharmacogenomics. 2002;3: 201-18.

12. de Vries E, Pot H, Conemans J, Uges D. The clinical relevance of routine genotyping of CYP2D6/2C19 null alleles of psychiatric patients. Br J Clin Pharmacol. 2002;53:550P-1P.

13. National Cancer Institute. Risks Associated With Smoking Cigarettes With Low Machine-Measured Yields of Tar and Nicotine. Smoking and Tobacco Control Monograph No. 13. Bethesda, Md: US Department of Health and Human Resources, National Institutes of Health, National Cancer Institute. NIH Pub. No. 02-5074, October 2001.

14. Lerman C, Hughes C, Croyle R, et al. Prophylactic surgery decisions and surveillance practices one year following BRCAl/ 2 testing. Prev Med. 2000;31:75-80.

15. Lerman C, Gold K, Audrain J, et al. Incorporating biomarkers of exposure and genetic susceptibility into smoking cessation treatment: effects on smoking-related cognitions, emotions, and behavior change. Health Psychol. 1997;16:87-99.

16. Audrain J, Boyd N, Roth J, Main D, Caporaso N, Lerman C. Genetic susceptibility testing in smoking-cessation treatment: one-year outcomes of a randomized trial. Addict Behav. 1997;22: 741-51.

17. Senior V, Marteau T, Peters T. Will genetic testing for predisposition for disease result in fatalism? A qualitative study of parents' responses to neonatal screening for familial hypercholesterolaemia. Soc Sci Med. 1999;48:1857-60.

18. Leventhal H, Safer M, Panagis D. The impact of communications on the self-regulation of health beliefs, decisions, and behavior. Health Educ Q. 1983;10:3-29.

19. McBride C, Bepler G, Lipkus I, et al. Incorporating genetic susceptibility feedback into a smoking cessation program for African-American smokers with low income. Cancer Epidemiol Biomarkers Prev. 2002;11:521-8.

20. Eddy D. Screening for lung cancer. Ann Intern Med. 1989;111: 232-7.

21. Marcus P, Bergstralh E, Fagerstrom R, et al. Lung cancer mortality in the Mayo Lung Project: impact of extended followup. J Natl Cancer Inst. 2000;92:1308-16.

22. Henschke C, McCauley D, Yankelevitz D, et al. Early Lung Cancer Action Project: overall design and findings from baseline screening. Lancet. 1999;354:99-105.

23. Heffner J, Silvestri G. CT screening for lung cancer: is smaller better? Am J Respir Crit Care Med. 2002;165:433-4.

24. Swenson S, Jett J, Sloan J, et al. Screening for lung cancer with low-dose spiral computed tomography. Am J Respir Crit Care Med. 2002;165:508-13.

25. Omenn G, Goodman G, Thornquist M, et al. Effects of a combination of beta carotene and vitamin A on lung cancer and cardiovascular disease. N Engl J Med. 1996;334:1150-5.

26. Mattson M, Pollack E, Cullen J. What are the odds that smoking will kill you? Am J Public Health. 1987;77:425-31.

27. Strachan D, Cook D. Health effects of passive smoking. 1. Parental smoking and lower respiratory illness in infancy and early childhood. Thorax. 1997;52:905-14.

28. Strachan D, Cook D. Parental smoking and childhood asthma: longitudinal and case-control studies. Thorax. 1998;53:204-12.

29. Anderson H, Cook D. Passive smoking and sudden infant death syndrome: review of the epidemiological evidence. Thorax. 1997; 52:1003-9.

30. Boffetta P, Agudo A, Ahrens W, et al. Multicenter case-control study of exposure to environmental tobacco smoke and lung cancer in Europe. J Natl Cancer Inst. 1998;90:1440-50.

31. Steenland K, Thun M, Lally C, Heath C. Environmental tobacco smoke and coronary heart disease in the American Cancer Society CPS-II cohort. Circulation. 1996;94:622-8. 
32. Cromwell J, Bartosch W, Fiore M, Hasselblad V, Baker T. Costeffectiveness of the clinical practice recommendations in the AHCPR guideline for smoking cessation. JAMA. 1997;278: 1759-66.

33. Cross F. Facts and values in risk assessment. Reliability Engineering and System Safety. 1998;59:27-40.
34. Wilson R, Crouch E. Risk assessment and comparisons: an introduction. Science. 1987;236:267-70.

35. Marteau T, Lerman C. Genetic risk and behavioural change. BMJ. 2001;322:1056-9.

36. Jemal A, Thomas A, Murray T, Thun M. Cancer statistics, 2002. CA Cancer J Clin. 2002;52:23-47.

\section{YOU'RE INVITED TO VISIT \\ The SGIM Website}

Portal \& Pathway

to Professional Effectiveness \& Satisfaction offering

Knowledge - Networking - Career Development

Featuring links to resources \& tools

including meetings, publications, job listings, funding sources, Residency \& Fellowship directories, government agencies,

$\&$ search engines

Located at http://www.sgim.org 